\title{
Research on the Influence of China's Urbanization Process on Rural Construction
}

\author{
${ }^{1}$ Zou Zaijin ${ }^{*}{ }^{2}$ Yang Lei \\ 1,2 School of Public Administration, \\ Yunnan University of Finance and Economics, \\ Kunming, Yunnan, P. R. China \\ (E-mail: 1 spa3238@163.com,2cn.youngly@foxmail.com)
}

\begin{abstract}
With the deepening of reform and opening up, the urbanization level in each region of China has improved quickly. The urbanization has caused the positive influence on the rural development, but the new rural problems are emerging in the process of urbanization. This paper defines the urbanization, analyzes the positive effect and negative effect of urbanization on rural construction based on the current situation of rural construction, and puts forward the direction of the rural construction in the urbanization process.
\end{abstract}

Keywords-China, Urbanization Process, Rural Construction

\section{INTRODUCTION}

China has been an agricultural country since the ancient times. Besides, the rural population accounts for a majority in the total populations, and the agricultural industry is an important part of national economy. The society is always developing in the direction of the high efficiency, so the second industry and the tertiary industry develop rapidly, but the proportion of agricultural production of the national economy is declining. At the same time, the rural population is moving to the town. With the development of industrialization and modernization, the urbanization has developed rapidly. Now, we should not only face the problems of backward rural economy, slow development, large rural population, but also face the new social problems in the process of rapid urbanization. In the report on $18^{\text {th }}$ National Congress of the $\mathrm{CCP}$, it proposed the new objective of building "beautiful China", so the economy, politics, culture, society and ecology should develop harmoniously. Building "beautiful country" is an important part of building "beautiful China", so in order to achieve the goal of building "beautiful China", the pace of rural construction must be accelerated. It is necessary to consider the effect of urbanization on rural construction when building the modern country in the process of urbanization.

\section{MEANING OF URBANIZATION AND DESTINATION OF RURAL CONSTRUCTION}

With the development of industrialization, the town will create more job opportunities compared to the country, so the agricultural population gradually moves to the town. Labor concentration is beneficial to the improvement on the industrial production efficiency and information level, which will produce the economies of scale. And with the increase in urban populations, the labor force will not only transfer to the second industry, but also gradually transfer to the third industry, so that the second and third industry will occupy more important position in the national economy, which is conducive to the adjustment and optimization of regional industrial structure, and helpful to speed up the development of the whole region. The rapid development of the town will continue to attract resources, such as the rural population, to the town[1].

China's "urbanization" focuses on the effect of small towns and central towns compared to the concept of "urbanization" abroad, which is manifested that the rural population transforms into the urban population and the towns develop and improve. At present, the urban areas in our country have developed rapidly and gathered a large number of resources, which produces obvious gaps between it and the slow development of rural areas. In order to radiate the most rural areas, provide sufficient support to the countryside and solve the rural problems, we have to speed up the development of small towns and central towns, improve the level of local economy and culture, absorb the appropriate resources, and make the small towns and central towns shoulder more social functions.

The urbanization is a process that the agricultural population transforms into the non-agricultural population, rural area transforms into the non-agricultural areas, and the agricultural activities transform into the non-agricultural activities.[2] It has the following features: first, the urban area is increasing, while the rural area is decreasing; second, more and more rural residents transform into the urban residents, and their production mode, living habits and collaboration form are changed; moreover, they should perform the duty of urban residents, enjoy the corresponding rights and social welfares, so the total number of farmers will decrease, and the cultural level and life quality of the residents will improve; third, the agricultural production will be conducted by industrialized way, which will greatly improve the efficiency of the first industry. The transfer of populations of the second and third industry will change the status of the second and third industry in the national economy, optimize the economic structure and promote the rapid economic development.

Based on the urbanization, it becomes the pursuit of China's modern rural construction to build modern new rural areas with livable ecological environment, comprehensive 
infrastructure, developed industrial economy, harmonious society, and diverse spiritual cultural life become.

\section{EFFECT OF URBANIZATION ON \\ BEAUTIFUl RURAL CONSTRUCTION}

\section{A. Positive effect}

Urbanization have gathered various factors of production resources, which can achieve the scale operation, use the modern production technology and production equipment, and greatly improve the efficiency of agricultural production.

The urbanization improves the roads, water conservancy, communications, energy and other infrastructure constructions of the rural areas.

Urbanization allow the good education, science and technology, business model and health care to benefit the rural areas, improve the scientific and cultural level, the legal sense and moral level of the rural population, so that the farmers can play a better role as the main body of the rural construction.

\section{B. Negative effect}

The rapid urbanization and industrialization causes the excessive resource exploitation, serious industrial pollution that is transferred to rural areas quickly.

The urbanization development needs a large number of urban lands and industrial lands, so a large number of agricultural lands are transformed into non-agricultural land, reducing the agricultural land and limiting the development of the agricultural industry[4].

In the process of urbanization abroad, most rural populations is transformed into the urban residents. Although the registered residence of rural residents has indeed become the urban residents, their production and life style have not been urban yet. At the same time, the urbanization makes farmers lose agricultural lands, but most farmers have no other production skills. Due to low education level and weak new skills learning ability of rural residents, their employment is very limited, and most can only choose the hard jobs with low salary, such as the public security and cleaner, which lacks the long-term stability and is difficult to support the family[5].

The urbanization pushes the rural surplus labor force to efficient towns, but the human resources of rural construction are insufficient. The elderly, children, those with lower education level and the disabled account for more, while the young and middle-aged labor forces are insufficient in rural areas. And the most rural cadres are middle-aged adults who lack the energy and vigor and hard to accept new things, lack the new ideas and innovative spirit, so it is hard for them to carry out the work[6].

In the process of rapid urbanization, the rural transformation is too extensive, so there are "mass-demolishing mass-construction" and "same appearance of thousand villages". Under the condition of imperfect laws and regulations, many traditional villages, streets, ancient buildings and historic sites are damaged. The carrier of traditional production modes of rural China, the traditional way of life and the traditional culture is disappearing quickly.

\section{Direction of RuRAL ReCONSTRUCTION IN THE URBANIZATION PROCESS}

\section{A. Government led and multi-agent act jointly}

The transformations of government functions of latest rural construction have been to pay more attention. Local governments should guide and coordinate government departments, villagers and village organizations, enterprises, and various social organizations by the planning and finance policy. Let each subject contribute according to its characteristic, to improve the quality and efficiency of rural construction.

\section{B. Industrialize agriculture}

The modern rural construction pursues the industrialization development of agriculture, and replaces the traditional agriculture with modern agriculture production modes. There are rich economic forms brought by the urbanization, so the rural areas can use a variety of economic forms to transform the traditional small-scale agricultural mode to the agricultural enterprise management. The government, villagers, enterprises and social organizations participate in it with many kinds of cooperation forms, so as to promote the standardization and the intensive management of agricultural production, ensure the product specification and quality, integrate the links to production and sales, form a complete industrial chain, realize the agricultural modernization and industrialization, improve the efficiency of agricultural production, improve the level of the rural economy and increase the farmers' income level.

\section{Diversify industries}

The urbanization reduces the cost of communication between urban and rural areas, shortens the distance between urban and rural areas, strengthens the connection between the urban and rural areas, which is advantageous to the new industry development of rural areas, form the diversified industrial development, reduce the risk of single industry, optimize the industrial structure and improve the local economic level. Besides, it can provide farmers with various jobs and entrepreneurial opportunities, solve the employment problem of the farmers, and help villagers adapt to the changes brought by the urbanization.

\section{Develop the ecological civilization in the rural modern development}

The rural construction will not slow down, and the investment in infrastructure, industrial workshop and agricultural machinery will be further increased, and the largescale transformation will not stop, so it is a huge challenge in the process of urbanization that how to minimize the environmental pollution, improve living environment, inherit the village, architecture, customs and culture, improve the rural ecological environment and living environment in the largescale modern development. 


\section{E. Cultivate new farmers}

At present, the farmers produce with the family, so the economic income is not high. The modern rural construction pursues the industrialization development of rural economy, so the high-quality labor forces are needed. However, the rural residents' scientific and cultural level is not high nowadays, and the superstition and conservative ideas still exist. The rural modern construction needs to improve the status situation, change the concept of farmers, improve the scientific and cultural level, professional and technical level, ideological and moral levels of farmers, improve the overall quality of farmers, and cultivate the new farmers who are intellectual, welleducated and polite with moral and technology ability and suitable for the industrial economy, which needs the government's guidance and whole society's joint efforts.

\section{F. Improve public services to ensure harmonious development}

To guarantee the harmonious development of rural areas, it is necessary to have a thorough understanding of the villagers' willingness and demand, perfect the rural democracy at the grassroots level, let the villagers express their own wills and decisions and participate in social management and social affairs; It is also necessary to establish the safe social environment, perfect social security system and provide good public services; And it is necessary to provide the comprehensive infrastructure facilities, good education, health care, perfect social security and other public products of the villagers. Improve public services, promote the free flow of factors and resources between urban and rural areas, allocate all kinds of resources efficiently between urban and rural areas, narrow the gap between urban and rural areas, improve the farmers' material life and spiritual life, to realize the overall development of urban and rural areas. At present, however, it still needed the whole society's exploration and efforts. ${ }^{[7]}$

\section{CONCLUSION}

Rural construction is the catalyst for the rapid development of rural China, and also the essential means to solve the rural problems, narrow the gap between urban and rural areas, and realize the integration of urban and rural development. In the process of rapid urbanization in China, there presents some new rural problems, thus, faced with various opportunities and challenges on the rural construction, we should seriously analyze the reality, constantly explore the solutions and find the proper development direction.

\section{REFERENCES}

[1] Cheng Shu. The Study of Rural Labors' Migration in the Process of Urbanization[D]. Harbin: Northeast Agricultural University, 2013. (In Chinese)

[2] Luo Yingguang. Special Urbanization Development Research of Yunnan[D]. Kunming: Yunnan University, 2012. (In Chinese)

[3] Zhang Xi. Ministry of Agriculture: the area of cultivated land degradation has been more than $40 \%$ in China (2014-12-17) http://news.youth.cn/gn/201412/t20141217_6283464.htm. (In Chinese)

[4] Liu Yansui, Zhou yang. Challenge and Solution of China' s Beautiful Rural Construction[J]. Journal of Agricultural Resources and Environment, 2015, 32(2): 97-105. (In Chinese)
[5] Huang Wenfu. Investigation report of educational work in Qibuchang Community[G]// Wang Xiaoping. Reflections on the problems of the integrated development in Chenggong urban and rural areas. Kunming: Yunnan people press, 2014: 283-284. (In Chinese)

[6] Liu Zhaozheng. Influence of urbanization on new rural construction[J]. Truth Seeking, 2013, 9: 84-88. (In Chinese)

[7] Yang Yi. A Study on China Rural-Urban Co-development Theory and Practices-Based on Economic Mechanism and Its Index System[D]. Chengdu: Southwestern University of Finance and Economics, 2013. (In Chinese) 